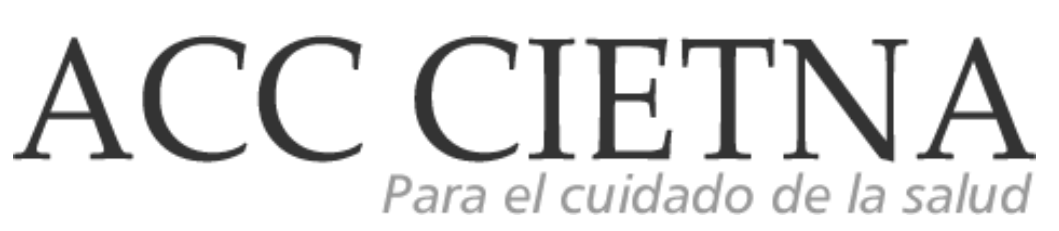

https://doi.org/10.35383/cietna.v8i2.604

e-ISSN: 2309-8570

Universidad Católica Santo Toribio de Mogrovejo

\title{
Factores de adherencia a la suplementación con multimicronutrientes en niños menores de 36 meses de Monsefú, 2020
}

\author{
Factores de adherencia a la suplementación con multimicronutrientes en \\ niños menores de 36 meses de Monsefú, 2020 \\ Noelia Melina Uceda-Vásquez $*$, 1, a \\ noeuceda31@gmail.com \\ https://orcid.org/0000-0002-7710-9173 \\ Maria Caridad Arriola-Arizaga ${ }^{1,2, a, b, c}$ \\ ${ }^{2}$ Centro de Salud de San José, Chiclayo, Perú \\ a Licenciada en Enfermería \\ b Magister en Bioética y Biojurídica \\ c Especialista en Salud Familiar y Comunitaria
}

marriola@usat.edu.pe

https://orcid.org/0000-0002-3524-1950

\author{
* Autor corresponsal \\ 1 Escuela de Enfermería, Facultad de Medicina, \\ Universidad Católica Santo Toribio de Mogrovejo, \\ Chiclayo, Perú
}

\author{
Fechas importantes \\ Recibido: 2021-05-27 \\ Aceptado: 2021-11-15 \\ Publicado online: 2021-12-17
}

\section{Resumen}

Objetivo: Determinar el grado de los factores de adherencia a la suplementación con multimicronutrientes en niños menores de 36 meses de Monsefú, 2020. Método: La población y muestra estuvo conformada por 100 madres de niños menores de 36 meses pertenecientes a la jurisdicción del Centro de Salud de Monsefú. El muestreo fue no probabilístico por conveniencia y la recolección de datos se realizó a través de una ficha de datos generales y el cuestionario realizado por Espichan. Los datos obtenidos fueron procesados de manera que se logró calcular frecuencias absolutas y medidas de tendencia central, además se midió la confiabilidad del instrumento utilizado mediante alfa de Cronbach. Durante el proceso de la investigación se cumplió con los criterios de rigor ético y científico. Resultados principales: El $97 \%$ de madres obtuvo un grado de adherencia 'regular'. La dimensión con mayor porcentaje de madres con adherencia alta la correspondiente a los factores del suplemento multimicronutriente (35\%). El mayor porcentaje de adherencia regular lo obtuvo la dimensión de los factores del personal de salud (99\%) y el mayor porcentaje de adherencia baja, la dimensión de los factores socioeconómicos (17\%). Conclusión general: La dimensión del personal de salud fue la que presentó mayor porcentaje de madres con adherencia regular, el factor más relevante 
fue su desconfianza frente a la información que el personal de salud les brinda, desencadenando una serie de factores que no contribuyen en el logro de un grado de adherencia alto en las 4 dimensiones restantes.

Palabras clave: Multimicronutriente, adherencia (D024362), suplementación (D019587), micronutriente (D018977), niños (D002648)

\begin{abstract}
Objective: Determines the degree of adherence factors to multi-micronutrient supplementation in children under 36 months of Monsefú, 2020. Method: The population and sample was made up of 100 mothers of children under 36 months who went at Monsefú's Health Center. The sampling was not probabilistic for convenience and the data collection was carried out through a fact sheet and the test carried out by Espichan. The data were processed and were calculated absolute frequencies and measures of central tendency, in addition the reliability of the instrument was measured throught Cronbach's alpha. During the research process, ethical and scientific criteria of rigour were met. Main results: That $97 \%$ of mothers obtain a degree of "regular" adherence. The dimension with the highest percentage of mothers with high adherence corresponds to the factors of the multimicronutrient supplement (35\%). The highest percentage of regular adherence was obtained by the dimension of the health personnel factors (99\%) and the highest percentage of low adherence, the dimension of socioeconomic factors (17\%). General conclusion: The health personnel dimension had the highest percentage of mothers with regular adherence, the most relevant factor being the mothers' distrust of the information provided by health personnel, triggering a series of factors that do not contribute to the achievement of a high degree of adherence in the remaining four dimensions.
\end{abstract}

Keywords: Multi-micronutrients, adherence (D024362), suplementation (d019587), micronutrients (d018977), child (d002648).

\title{
Introducción
}

Una nutrición inadecuada en los tres primeros años de vida del niño puede traer consigo la carencia de nutrientes $y$, como resultado, enfermedades asociadas a ella que puede comprometer la maduración del sistema nervioso, visual, mental e intelectual ${ }^{1}$. Por ello, la suplementación con multimicronutrientes (MMN), ha sido implementada en 40 países alrededor del mundo siendo la intervención estratégica más efectiva en casos de déficit nutricional en niños, debido a su alta aceptabilidad, su fácil uso dentro del entorno alimentario familiar y a su gran aporte nutricional en la alimentación diaria ${ }^{2,3}$.

En países de Latinoamérica, existen diversos programas encargados de la administración, monitoreo y seguimiento de la suplementación con MMN. Sin embargo, la inversión realizada por cada uno de los gobiernos solo ha permitido mantener la cobertura actual sin poder aumentarla como se desea, evidenciándose la incidencia de anemia y retraso del crecimiento en niños(as), como resultado de la deficiencia de hierro (más del 50\%), vitamina A (23-30\%.) y zinc (30-50\%), las mismas que sumadas a la deficiencia de vitamina $C$ y ácido fólico, conllevan a enfermedades como, ceguera, inmunodeficiencia, coagulopatías, entre otras $4,5,6$.

A todo esto, en países de Latinoamérica se reconoció que la adherencia a esta suplementación es condicionada principalmente por factores relacionados al personal de salud encargado, como también de la persona a cargo de la administración del MMN, pues cumple con la función de proporcionar el suplemento y garantizar su efectividad ${ }^{1}$. Por una parte, los factores que influyen en el personal de salud son, la dificultad en el acceso y el sistema de distribución a la población, problemas en la adquisición, distribución, monitoreo, control de calidad, almacenamiento de los MMN y las limitaciones en el punto de entrega. Por otra parte, los 
factores de la persona que está a cargo de la administración de MMN son, el conocimiento de los beneficios de los MMN, la predisposición de los padres/cuidadores y, la preparación y administración adecuada de los MMN al niño ${ }^{1,6}$.

Rojas y Suqui ${ }^{7}$ se inclinan por los factores relacionados a la persona encargada de la suplementación con MMN, aseverando que, el $40 \%$ de madres ecuatorianas tiene conocimientos adecuados, el $73 \%$ se muestra positiva al momento de administrar los MMN y el 39\% tiene una excelente práctica, concluyendo que el $40 \%$ de las madres tienen nociones acerca de las utilidades y consecuencias de la suplementación de MMN.

En Perú, los factores de la problemática en mención se reparten en 5 dimensiones, tenemos: dimensión socioeconómica, dimensión del personal de enfermería, dimensión del suplemento MMN, dimensión la persona a cargo de la administración del MMN y dimensión de enfermedad ${ }^{8}$.

Espichán ${ }^{9}$, por su parte, expone que el $41 \%$ de encuestados interrumpió la suplementación con MMN en sus hijos(as), debido a infección respiratoria aguda, siendo un total de $64 \%$ de niños(as) con adherencia alta. Asimismo, Hinostroza $^{10}$ en su investigación obtuvo que, el $8.5 \%$ de madres alcanzó un grado alto de adherencia, mientras que la del $91.5 \%$ fue baja, indicando que, a pesar de la presencia de efectos adversos por el consumo de MMN, las madres observaron beneficios en sus hijos(as). El factor olvido predominó en las madres. Por último, en el estudio de Pesantes ${ }^{11}$ se enfatizó que los factores socioeconómicos influyen en la suplementación con MMN, siendo prioridad la edad y el grado de escolaridad, y dentro de los factores del personal de salud predomina la demora y la calidad de atención.

Frente a la problemática expuesta, esta investigación tuvo como objetivo general: Determinar el grado de los factores de adherencia a la suplementación con multimicronutrientes en niños menores de 36 meses de Monsefú. Los objetivos específicos son identificar el grado de los factores de adherencia, según la dimensión socioeconómica, según la dimensión del personal de salud, según la dimensión del suplemento multimicronutriente, según la dimensión de la persona que administra el multimicronutriente y según la dimensión enfermedad en niños menores de 36 meses.

La presente investigación se justifica en base a información de diversas fuentes y antecedentes que demuestran la persistencia de la inadherencia a la suplementación con MMN debido a factores que influyen negativamente en ella, ocasionando repercusiones a nivel psicomotor, cognitivo y físico en la salud infantil, la que actualmente se ve afectada debido a la alta tasa de morbimortalidad en enfermedades prevenibles con el consumo de MMN ${ }^{12,13}$. Además, se conoce que en nuestro país, la alimentación diaria brindada por la mayoría de madres a sus hijos(as) es de bajo aporte nutricional, razón por la cual es fundamental abordar los factores que no favorezcan la adherencia a la suplementación con MMN e implementar intervenciones para el logro de una mayor adherencia en las madres $y$, de esta forma, contribuir en el mejoramiento de la salud de sus hijos(as) ${ }^{14}$.

\section{Material y métodos}

Se realizó un estudio piloto ${ }^{15,16,17,18}$ con enfoque cuantitativo ${ }^{19}$ descriptivo ${ }^{20,21}$. La población y muestra fue conformada por 100 madres de niños menores de 36 meses que recibieron MMN en el Centro de Salud de Monsefú. Dicho número de participantes fue encuestado en febrero del 2020 antes de que el ex-presidente de la República, Martín Vizcarra Cornejo, declarara aislamiento social obligatorio debido al Estado de Emergencia en nuestro país ${ }^{22}$. El tipo de 
muestreo fue no probabilístico, por conveniencia ${ }^{15}$. Se incluyeron a madres cuyos hijos hayan sido suplementados con MMN durante el año 2019, en un periodo no menor de 3 meses, pertenecientes a la jurisdicción del Centro de Salud de Monsefú y que por voluntad propia hayan aceptado participar de la investigación. Por otro lado, se excluyeron a aquellas madres que presenten alguna discapacidad para responder el cuestionario o que sean menores de 18 años y no se encuentren acompañadas de una persona adulta.

Se utilizó una ficha de datos generales y el Test de Adherencia a la Suplementación con Multimicronutrientes realizado por Espichan ${ }^{9}$, que fue medido con el coeficiente alfa de Cronbach, obteniendo un $a=0,72$ y fue validado por su autor a través de juicio de expertos para verificar la coherencia entre las preguntas y las variables, con sus respectivas dimensiones, obteniendo un $100 \%$ por parte de los jueces comprobando que los ítems que constituyen el instrumento tienen el dominio del contenido que se mide ${ }^{23}$.

Dicho instrumento está basado en las 5 dimensiones de los factores de adherencia, haciendo un total de 23 preguntas, las mismas que presentan 3 alternativas de puntuación: adherencia baja (1 punto), adherencia regular ( 2 puntos) y adherencia alta ( 3 puntos). Con el fin de clasificar el puntaje por dimensión de cada participante, se debe tener en cuenta que en la dimensión socioeconómica y la dimensión del MMN, se considera adherencia alta cuando se obtiene entre 13-15 puntos, de 8-12 es adherencia regular y de 5-7 es adherencia baja. En cuanto a la dimensión del personal de salud, de 11-12 puntos se trata de adherencia alta, de 6-10 puntos adherencia regular y de 4-5 puntos adherencia baja. Respecto a la dimensión de la persona que administra el MMN, una persona presenta adherencia alta cuando su puntaje varía entre 18-21 puntos, adherencia regular cuando se encuentra entre 11-17 puntos y adherencia baja cuando esta entre 7-10 puntos. En relación a la dimensión de enfermedad, se obtiene adherencia alta con 6 puntos, adherencia regular con 4-5 puntos y adherencia baja con 2-3 puntos. Por último, para clasificar el puntaje total, se trata de adherencia alta cuando la persona obtuvo un puntaje total de 57-69 puntos, de adherencia regular cuando el puntaje fue 33-56 puntos y de adherencia baja cuando fue de 23-32 puntos.

El proyecto recibió la aprobación del Comité de Ética de Investigación de la Facultad de Medicina de la Universidad Católica Santo Toribio de Mogrovejo con resolución N654-2019USAT-FMED. Por consiguiente, se solicitó permiso al Gerente del Centro de Salud de Monsefú para su ejecución, con el que se hizo la coordinación respectiva con la coordinadora de Crecimiento y Desarrollo ${ }^{23}$. Posteriormente, se mantuvo un primer acercamiento con las madres usuarias para brindarles información detallada acerca de su participación y se procedió a la ejecución del proyecto y la recolección de datos. Seguidamente, se hizo entrega del cuestionario para el desarrollo por parte de las participantes

El procesamiento de datos se realizó mediante una base de datos en el programa SPSS versión 25 y en Microsoft Excel. Así mismo, se calcularon frecuencias absolutas y medidas de tendencia central, para la posterior elaboración de tablas y gráficos. Se calculó el Alfa de Cronbach de las 23 preguntas que conforman el instrumento para verificar su consistencia interna obteniendo $0.069^{24}$.

Durante la ejecución, se tuvo en cuenta los principios bioéticos de Sgreccia. Se respetó el principio de libertad y responsabilidad ya que las madres participaron libremente en el estudio, después de firmar la hoja informativa entregada por la investigadora ${ }^{25}$. Además, se respetó el principio de sociabilidad y subsidiariedad ya que se logró la participación activa de las madres, permitiéndoles contribuir en el bien común de la investigación: promover el buen estado 
nutricional de los niños de Monsefú y evitar el desarrollo de enfermedades prevenibles con el consumo de $\mathrm{MMN}^{26}$.

\section{Resultados}

Tabla No 01

Grado de los factores de adherencia a la suplementación con MMN en niños menores de 36 meses de Monsefú, 2020

\begin{tabular}{lcc} 
Grado de Adherencia & Frecuencia (N) & Porcentaje (\%) \\
\hline Alto & 3 & $3 \%$ \\
Regular & 97 & $97 \%$ \\
Bajo & 0 & $0 \%$ \\
\hline TOTAL & $\mathbf{1 0 0}$ & $\mathbf{1 0 0 \%}$
\end{tabular}

Fuente: Elaboración propia

\section{Interpretación:}

El grado de los factores de adherencia a la suplementación con multimicronutrientes en niños menores de 36 meses fue regular en el $97 \%$ del total de madres encuestadas.

Tabla No 02

Grado de adherencia a la suplementación con multimicronutrientes, según edad de la madre

\begin{tabular}{|c|c|c|c|c|c|c|c|c|}
\hline \multirow{3}{*}{$\begin{array}{l}\text { Grado de } \\
\text { adherencia }\end{array}$} & \multicolumn{8}{|c|}{ Edad de la madre } \\
\hline & \multicolumn{2}{|c|}{15 -25 años } & \multicolumn{2}{|c|}{$26-35$ años } & \multicolumn{2}{|c|}{$36-45$ años } & \multicolumn{2}{|c|}{ Total } \\
\hline & $\mathbf{N}$ & $\%$ & $\mathbf{N}$ & $\%$ & $\mathbf{N}$ & $\%$ & $\mathbf{N}$ & $\%$ \\
\hline Alta & 1 & $1 \%$ & 1 & $1 \%$ & 1 & $1 \%$ & 3 & $3 \%$ \\
\hline Regular & 19 & $19 \%$ & 52 & $52 \%$ & 26 & $26 \%$ & 97 & $97 \%$ \\
\hline TOTAL & 20 & $20 \%$ & $\begin{array}{r}53 \\
\text { nte: }\end{array}$ & $\begin{array}{l}53 \% \\
\text { boraci }\end{array}$ & $\begin{array}{c}27 \\
\text { n pro }\end{array}$ & $27 \%$ & 100 & $100 \%$ \\
\hline
\end{tabular}

\section{Interpretación:}

Del $97 \%$ de madres con grado de adherencia regular, el 52\% presenta una edad entre $26-35$ años, el $26 \%$ se encontraba entre $36-45$ años y, por último, el $19 \%$ de madres con edad entre 15-25 años. El porcentaje de madres con adherencia alta (3\%), se divide en los 3 rangos de edad. 
Tabla No 03

Grado de adherencia a la suplementación con multimicronutrientes, según edad de la madre

Grado de instrucción de la madre

\begin{tabular}{|c|c|c|c|c|c|c|c|c|c|c|c|c|c|c|}
\hline \multirow[t]{2}{*}{$\begin{array}{l}\text { Grado de } \\
\text { adherencia }\end{array}$} & \multicolumn{2}{|c|}{$\begin{array}{l}\text { Educación } \\
\text { superior }\end{array}$} & \multicolumn{2}{|c|}{$\begin{array}{l}\text { Educación } \\
\text { técnica }\end{array}$} & \multicolumn{2}{|c|}{$\begin{array}{l}\text { Primaria } \\
\text { completa }\end{array}$} & \multicolumn{2}{|c|}{$\begin{array}{l}\text { Primaria } \\
\text { incompleta }\end{array}$} & \multicolumn{2}{|c|}{$\begin{array}{l}\text { Secundaria } \\
\text { completa }\end{array}$} & \multicolumn{2}{|c|}{$\begin{array}{l}\text { Secundaria } \\
\text { incompleta }\end{array}$} & \multicolumn{2}{|c|}{ Total } \\
\hline & $\mathbf{N}$ & $\%$ & $\mathbf{N}$ & $\%$ & $\mathbf{N}$ & $\%$ & $\mathbf{N}$ & $\%$ & $\mathbf{N}$ & $\%$ & $\mathbf{N}$ & $\%$ & $\mathbf{N}$ & $\%$ \\
\hline Alta & 1 & $1 \%$ & & & 1 & $1 \%$ & & & 1 & $1 \%$ & & & 3 & $3 \%$ \\
\hline Regular & 12 & $12 \%$ & 17 & $17 \%$ & 19 & $19 \%$ & 7 & $7 \%$ & 27 & $27 \%$ & 15 & $15 \%$ & 97 & $97 \%$ \\
\hline Total & 13 & 10 & 17 & $\%$ & 20 & $\begin{array}{l}20 \\
\%\end{array}$ & 7 & & 28 & 0 & 15 & $15 \%$ & 100 & $100 \%$ \\
\hline
\end{tabular}

Interpretación:

Del $97 \%$ de madres con adherencia regular, las que recibieron secundaria completa representa a la mayoría con un $28 \%$. Por otro lado, las madres que percibieron educación técnica $(17 \%)$ y superior (13\%) abarcan el $30 \%$ y aquellas que presentan primaria incompleta $(7 \%)$, primaria completa $(20 \%)$ y secundaria incompleta $(15 \%)$ son representadas por el $42 \%$. En relación al $3 \%$ de madres con adherencia alta, dicho porcentaje incluye al $1 \%$ de madres con educación superior, el $1 \%$ con primaria completa y, por último, el $1 \%$ con secundaria completa.

\section{Tabla No 04}

Grado de los factores de adherencia a la suplementación con $\mathrm{MMN}$, según dimensiones, en niños menores de 36 meses de Monsefú, 2020

\begin{tabular}{|c|c|c|c|}
\hline Factores & $\begin{array}{c}\text { Grado de } \\
\text { adherencia }\end{array}$ & $\begin{array}{l}\text { Frecuencia } \\
\text { (N) }\end{array}$ & $\begin{array}{c}\text { Porcentaje } \\
(\%)\end{array}$ \\
\hline \multirow{4}{*}{ Dimensión socioeconómica } & Alto & 4 & $4 \%$ \\
\hline & Regular & 79 & $79 \%$ \\
\hline & Bajo & 17 & $17 \%$ \\
\hline & TOTAL & 100 & $100 \%$ \\
\hline \multirow{4}{*}{ Dimensión personal de salud } & Alto & 1 & $1 \%$ \\
\hline & Regular & 99 & $99 \%$ \\
\hline & Bajo & 0 & $0 \%$ \\
\hline & TOTAL & 100 & $100 \%$ \\
\hline \multirow{4}{*}{ Dimensión del MMN } & Alto & 35 & $35 \%$ \\
\hline & Regular & 65 & $65 \%$ \\
\hline & Bajo & 0 & $0 \%$ \\
\hline & TOTAL & 100 & $100 \%$ \\
\hline \multirow{4}{*}{$\begin{array}{l}\text { Dimensión de la persona } \\
\text { que administra el MMN }\end{array}$} & Alto & 7 & $7 \%$ \\
\hline & Regular & 93 & $93 \%$ \\
\hline & Bajo & 0 & $0 \%$ \\
\hline & TOTAL & 100 & $100 \%$ \\
\hline
\end{tabular}




\begin{tabular}{llll}
\hline \multirow{3}{*}{ Dimensión de enfermedad } & Alto & 15 & $15 \%$ \\
& Regular & 69 & $69 \%$ \\
\cline { 2 - 4 } & Bajo & 16 & $16 \%$ \\
\cline { 2 - 4 } & TOTAL & $\mathbf{1 0 0}$ & $\mathbf{1 0 0 \%}$ \\
\hline
\end{tabular}

Fuente: Elaboración propia

\section{Interpretación:}

El grado de los factores de adherencia, según la dimensión socioeconómica en la mayoría (79\%) de madres es regular. Según la dimensión del personal de salud, fue regular con el $99 \%$ del total de encuestadas. Respecto al grado de adherencia en la dimensión del suplemento multimicronutriente fue regular con el $65 \%$. El $93 \%$ obtuvo un grado de adherencia regular en relación al grado de adherencia según la dimensión de la persona que administra el multimicronutriente. Por último, el resultado del grado de los factores de adherencia, según la dimensión enfermedad fue regular evidenciándose en el $69 \%$ del total de madres.

\section{Discusión}

El grado de adherencia total a la suplementación con MMN que la mayoría de madres presentó fue regular $(97 \%)$, resultado que discrepa tras ser contrastado con los resultados de Hinostroza ${ }^{10}$, puesto que las participantes de su estudio no presentaron adherencia regular; por el contrario, percibieron baja adherencia a los MMN (91.5\%). Así mismo, se encuentra un estudio que midió los factores de adherencia a la suplementación con MMN en menores de 60 meses $^{9}$, con el que existe discrepancia ya que solo el $30 \%$ de madres calificaron con un grado de adherencia regular.

Cabe mencionar que el rol que ejercen los padres y/o cuidadores es una condición básica en el crecimiento y desarrollo de los niños menores de 36 meses ya que la conducta que asuman durante el cuidado del niño(a) dependerá del conocimiento que ellos adquieran, específicamente en cuanto a la alimentación ${ }^{27}$. Por tal razón, se consideró relevante enlazar las variables edad y nivel educativo de las madres con los resultados obtenidos del grado de adherencia total a la suplementación con $\mathrm{MMN}$, debido a que dichos datos permiten caracterizar a las madres y, además, se considerarían aspectos más importantes para analizar, debido a que la educación influye en la salud familiar y, principalmente en la de los niños(as) ${ }^{28}$.

De los datos generales de la investigación se obtuvo que la edad de la mayoría de madres (53\%) varía entre $26-35$ años y de dicho porcentaje el $98.11 \%$ (52 madres) presenta adherencia regular, siendo solo el 1.88\% (1 madre) el porcentaje que obtuvo adherencia alta. Relacionar la edad con el grado de adherencia total permite predecir patrones de adherencia, siendo las madres adolescentes y jóvenes (15-25 años) las que menos se adhieren, reflejando cierto grado de resistencia frente al control del régimen de sus vidas y la de sus hijos(as); mientras que las madres adultas (26-59 años) presentan una mayor adherencia ${ }^{29}$. Estos resultados concuerdan con los de un estudio realizado en Etiopía ${ }^{30}$.

Respecto al nivel educativo, el $58 \%$ de madres percibe un mayor nivel educativo (secundaria completa y educación técnica -superior); mientras que el $42 \%$ percibe menor nivel educativo (primaria incompleta, primaria completa y secundaria incompleta). Al relacionar estos resultados con el grado de adherencia total resulta que, del porcentaje de madres con mayor nivel educativo, el $96.55 \%$ (56 madres) presentó adherencia regular y el porcentaje restante, 
adherencia alta. Por otro lado, del porcentaje de madres con menor nivel educativo, el $97.61 \%$ (41 madres) obtuvo adherencia regular y solo el $1 \%$ obtuvo adherencia alta. Esto permite identificar si las madres con determinado nivel educativo se han visto favorecidas tras la comprensión de la información brindada por el personal de enfermería acerca de la suplementación con MMN y si dicha información les permitió desarrollar un proceso de adaptación que favorezca la adherencia ${ }^{29}$.

Es importante tener en cuenta que el concepto del personal de salud, es que mientras mayor sea el nivel educativo de los padres de familia, mayor será la adquisición de conocimientos, destrezas, capacidades, actitudes y valores que le permitan lograr un mayor compromiso y grado de adherencia ${ }^{30}$. Sin embargo, en el estudio de Ibarra y Morillo ${ }^{29}$, los resultados respecto al nivel educativo fueron paradójicos pues se asevera que las personas con un alto nivel educativo cuestionan más las indicaciones dadas por el personal de salud y presentan un grado de adherencia más bajo (40\%). En contraposición, aquellas con nivel educativo más bajo mostraron valores más elevados de adherencia (55,9 \%).

En relación a la dimensión de los factores del personal de salud, el 99\% de madres perciben una adherencia regular, resultado que coincide con el $80 \%$ de madres con adherencia regular de un estudio realizado en $\operatorname{Lima}^{9}$ y con $78.3 \%$ de otra investigación ${ }^{31}$.

Este resultado muestra que el personal de salud no cumple por completo las expectativas de atención que las madres esperan tener, evidenciándose cierta dificultad para el logro de un alto grado de adherencia en esta dimensión. Para obtener tal resultado, debe generarse en ellas una mayor confianza hacia la suplementación con MMN ofreciendo un servicio de calidad, poniendo en práctica la escucha activa y la comunicación, habilidades que favorezcan la relación enfermera - paciente, el interés mostrado por el personal de salud y, principalmente, el entendimiento que las madres lleguen a demostrar respecto a la información brindada. Lo último, es de suma importancia puesto que, el $61 \%$ de madres asegura tener una regular confianza respecto a lo que el personal de salud les informa sobre los MMN.

Mayca, Medina, Velásquez y Llanos ${ }^{32}$, indican que la dificultad para adherirse a los MMN, además de basarse en las determinantes sociales como las creencias y costumbres de una comunidad, se ve influenciada por la manera en la que el personal de salud realiza la sensibilización a las madres en cuanto a la adherencia a la suplementación con MMN. Pues, este debe brindar información verbalmente manteniendo el contacto visual, demostrando evidencias palpables de los beneficios del consumo de MMN y reforzando constantemente a las madres en cada consulta a través de sesiones educativas y demostrativas, con el fin de aumentar la confianza, generar una mayor aceptabilidad y adherencia de las madres frente a los MMN, y lograr la satisfacción total respecto a la atención recibida ${ }^{11}$.

En contraste con una investigación realizada en Bangladesh ${ }^{33}$, existe concordancia con respecto a que el personal de salud debe promover intervenciones para el mejoramiento de la cobertura por parte de los establecimientos de salud, al igual que de la calidad de atención y el cumplimiento de la suplementación con MMN para el logro de una adherencia adecuada y un mayor control de enfermedades causadas por la deficiencia de micronutrientes en niños.

La dimensión de los factores relacionados al suplemento MMN fue en donde el $65 \%$ de madres calificó con un puntaje de adherencia regular, encontrando similitud con la investigación perteneciente a Ildefonso y Uturunco ${ }^{34}$, donde se obtuvo que el grado de adherencia más representativo de esta dimensión es el regular (68.6\%). Inclusive, en otra investigación se 
halló que el $50 \%$ de participantes percibía adherencia regular a los factores de esta dimensión ${ }^{31}$.

El $41.7 \%$ de madres en un estudio 35 informaron que sus hijos se vieron beneficiados con la administración de $\mathrm{MMN}$, rescatando: el aumento de energía, aumento de apetito, crecimiento físico y mayor desarrollo mental. Sin embargo, existen en su mayoría estudios con participantes que refieren presenciar en sus hijos efectos adversos que los conllevan a suspender la suplementación con MMN. Tal es el caso del presente estudio, siendo lo más resaltante en esta dimensión el $67 \%$ de madres que refirió haber notado, regularmente, efectos secundarios (estreñimiento, diarrea u otra molestia) en sus hijos(as). Otras investigaciones aseveraron lo mismo, dentro de ellas la de López $^{36}$ y Carrión ${ }^{37}$, donde el $56,7 \%$ y $76.6 \%$, respectivamente, afirmaron que presenciaron de forma regular los siguientes efectos secundarios: estreñimiento, pérdida de apetito y diarrea. Así mismo, en un estudio realizado en Inkawasi ${ }^{38}$, los efectos secundarios más recurrentes fueron la diarrea y el estreñimiento. Igualmente, en una investigación realizada en Brasil ${ }^{39}$ donde el efecto secundario principal fue la diarrea (41.7\%).

En la investigación realizada por Fernándes y Cardoso ${ }^{40}$, las madres además de reafirmar los mismos efectos en sus hijos, además incluyeron los cambios en el color o el sabor de los alimentos: el amarilleo del arroz, el amargor de los alimentos y el sabor desagradable después de mezclarlos con MMN. Sumado a ello en otra investigación ${ }^{41}$, indican como un efecto adverso el dolor abdominal tras el consumo de MMN.

La aparición de efectos secundarios por el consumo de MMN se debe a la inadecuada preparación de los mismos. Si durante este proceso los MMN son mezclados cuando el alimento está caliente y, además, su consistencia es líquida o semilíquida; como resultado, su sabor, olor, consistencia y color se verán alterados provocando síntomas a nivel gastrointestinal ${ }^{14}$. Por tanto, el que las madres de esta investigación presencien efectos secundarios en sus hijos tras el consumo de MMN, indica que existe una preparación inadecuada del suplemento, a pesar de considerar fácil su preparación. Este es el motivo por el cual los participantes con adherencia regular a esta dimensión no logran obtener una adherencia alta.

De acuerdo a los factores de la dimensión de la persona que administra el multimicronutriente, el $93 \%$ de madres obtuvieron un grado de adherencia regular. Este resultado se asemeja a un estudio hecho en $\mathrm{Lima}^{34}$, en donde demostraron que el $99.3 \%$ presentó adherencia regular. Sin embargo, se contrapone a un estudio realizado en el año 2018 , en el cual obtuvieron solo el $35 \%$ de madres con adherencia regular respecto a esta dimensión, ya que la mayoría obtuvo un grado de adherencia alta ${ }^{31}$.

El factor más recurrente en esta dimensión es el olvido, representado por el $67 \%$ de madres que olvidaron regularmente administrar los MMN a sus hijos(as). En un estudio realizado en Apurimac $^{42}$, el factor olvido predominó solo en el $6.2 \%$ de madres al igual que en un estudio hecho en $\mathrm{Nepal}^{35}$, donde el $11.6 \%$ de madres olvidó administrar MMN a sus hijos(as). En un estudio $^{43}$, las madres de Puno manifestaron que, debido a sus actividades domésticas y laborales, se olvidaban de administrar los MMN a sus hijos(as) además, muchas suponían que la administración de este suplemento solamente se realizaba en el horario del almuerzo, por lo que al momento de recordar que no le dieron el MNN a su niño(a), esperaban hasta el día siguiente. 
En la dimensión de los factores socioeconómicos, el 79\% de las madres alcanzó un grado de adherencia regular a la suplementación con MMN. Dicho resultado se iguala al del estudio realizado por Mamani ${ }^{44}$, en el cual el mayor porcentaje de madres (48.78\%) presentaron un grado regular de adherencia respecto a los factores socioeconómicos. Además, existe similitud con el resultado obtenido en una investigación realizada en Huancavelica ${ }^{31}$, con el $68,3 \%$ de madres que presentó una adherencia regular a la misma dimensión.

Además, en una investigación cualitativa realizada por Aparco y Huamán ${ }^{43}$, las madres que participaron manifiestan no sentirse motivadas ni apoyadas en la suplementación con MMN debido a la oposición presentada por sus esposos quienes adoptaron la creencia de que este suplemento impedía que sus hijos(as) crecieran y desarrollaran normalmente. Lo mismo sucedió con las madres y suegras de las participantes, quienes refirieron haber criado a sus hijos(as) sin ningún tipo de suplemento, por lo que no consideraban necesaria la administración de MMN a sus nietos(as). A consecuencia de la desmotivación presentada por las madres participantes del estudio, la adherencia a la suplementación con MMN se ve afectada.

Por lo expuesto, la motivación brindada por la familia es un factor socioeconómico fundamental para impulsar a las madres a lograr una mayor adherencia al consumo de MMN a través de su responsable administración a los niños(as). Sin embargo, en la presente investigación se evidencia que el $47 \%$ de madres manifestaron haber recibido regular motivación en casa, por parte de los integrantes de su familia. Esto se debe al escaso involucramiento de los padres en la crianza de los niños(as) debido a que la población aún mantiene un modelo estereotipado de las mujeres en el que únicamente deben desempeñar actividades domésticas mientras que los hombres, actividades laborales. También es notable la influencia de las abuelas en la toma de decisiones de los padres encargados de administrar los MMN, tendiendo a desistir de la suplementación y disminuir su grado de adherencia.

Con respecto a la última dimensión, de los factores relacionados a enfermedad, resultó que el grado de adherencia con mayor preponderancia es el regular, representado por el $69 \%$ del total de madres encuestadas. En contraposición, existe otro estudio en el que esta dimensión percibe el mayor porcentaje de adherencia baja $(12 \%)^{9}$.

El aspecto más predominante en esta dimensión es el regular conocimiento de las madres en cuanto a los signos y síntomas de anemia, representado por el $53 \%$ del total. En Tacna ${ }^{36}$, que obtuvo como resultado que el $56.4 \%$ de madres reconoce entre $1-2$ síntomas de anemia, considerándose como un conocimiento regular. Además, el resultado en Huanca Huanca ${ }^{31}$ fue similar con el $53.3 \%$ de madres que calificaron con adherencia regular.

En el año $2016^{33}$, además de la deficiencia de hierro en niños de edad preescolar (10.7\%), los autores identificaron otras deficiencias muy frecuentes, incluyendo la deficiencia de vitamina A $(20,5 \%)$, Zn $(44,5 \%)$ y vitamina D $(39,6 \%)$. Las deficiencias de micronutrientes interfieren en el desarrollo de los niños y generan una alta morbilidad en esta etapa de vida, siendo la enfermedad más frecuente, la anemia ${ }^{45}$.

La posición de la investigadora es que el grado de adherencia total a la suplementación con MMN, se ve afectado a causa de los factores más predominantes y especificados a lo largo del presente acápite, ya que interfieren en el logro de un grado de adherencia alto en cada una de las dimensiones debido a que todos los factores se relacionan entre sí. Por consiguiente, el mayor porcentaje de participantes percibe un grado de adherencia total regular, lo que 
significa que debe trabajarse en todas las dimensiones de manera igualitaria, pues la adherencia total no se modificará hasta que en cada una de sus dimensiones solo existan factores que faciliten la obtención de un grado de adherencia alto.

Finalmente, cabe mencionar que para la realización de este estudio se presentaron dificultades como el poco acceso a información relacionada a las 5 dimensiones de adherencia, en particular acerca de la dimensión enfermedad y socioeconómica, debido a que en la mayoría de las investigaciones mencionan los porcentajes de adherencia sin describir ni profundizar en los factores que influyen en ella. Sin embargo, tras una exhaustiva búsqueda bibliográfica se pudo obtener información mínima, pero de gran aporte para el estudio.

\section{Conclusiones}

El grado de adherencia total en el presente estudio fue regular, aportando el análisis de un panorama general que permitió identificar los factores que favorecieron y perjudicaron la adherencia a la suplementación con $M M N$ durante el último año que se estuvieron proporcionando, de modo que cuando se reanude su distribución sirva de orientación para el personal de salud, que deberá mejorar sus intervenciones para lograr una adherencia alta.

La dimensión del personal de salud fue la que presentó mayor porcentaje de madres con adherencia regular, siendo el factor más relevante la desconfianza de las madres frente a la información que el personal de salud les brinda, desencadenando una serie de factores que no contribuyen en el logro de un grado de adherencia alto en las dimensiones restantes.

Dentro de la serie de factores que no favorecen el aumento de la adherencia en las 4 dimensiones restantes, tenemos que en la dimensión del suplemento MMN, se encontró como principal factor a los efectos adversos tras el consumo de MMN, los que mayormente se presentan en el niño cuando el suplemento no ha sido preparado adecuadamente. También, el desconocimiento de los signos y síntomas de anemia por parte de los padres, es otro de los factores prioritarios en la dimensión de enfermedad. En la dimensión socioeconómica, predominó el factor social relacionado a la motivación en casa, en donde el mayor porcentaje de madres reportó sentirse desmotivadas regularmente. Por último, el factor olvido, en la dimensión de la persona que administra el MMN, es el más relevante ya que, al compararlo con otros estudios, existe una diferencia notable respecto al porcentaje de incidencia del olvido de la administración de MMN en las madres.

\section{Referencias}

1. Pereira $R$, Mendes $M$, de Pinho, L. Percepção dos pais sobre a implantação da estratégia de fortificação da alimentação infantil com micronutrientes. Rev. bras. promoç saúde [Internet]. Septiembre 2016 [Consultado 23 Dic 2020]; 29(3): 431-438. Disponible en: https://periodicos.unifor.br/RBPS/article/view/4539

2. De-Regil L, Suchdev P, Vist G, Walleser S, Peña J. Home fortification of foods with multiple micronutrient powders for health and nutrition in children under two years of age. Cochrane Database Syst Rev [Internet]. 09 agosto 2011 [Consultado 23 Dic 2020]; 8(1): 112-201. Disponible en: https://www.cochranelibrary.com/cdsr/doi/10.1002/14651858.CD008959.pub2/full

3. Suchdev P, Addo O, Martorell R, Grant F, Laird R, Patel M, et al. Effects of community-based sales of micronutrient powders on morbidity episodes in preschool children in Western Kenya. Am. J. Clin. Nutr. [Internet]. Marzo 2016 [Consultado 25 Abr 2020]; 103(3): 934-41. Disponible en: https://www.ncbi.nlm.nih.gov/pmc/articles/PMC4845747/

4. López D, Cediel G. Situación actual de los micronutrientes en Latinoamérica y el Caribe: Prevalencia de su deficiencia y programas nacionales de entrega de multimicronutrientes. En: Programa Mundial de Alimentos. Promoción de la fortificación del arroz en América Latina y el Caribe 
[Internet]. Panamá: Sight and life; 2015 [Consultado 02 Jun 2019]. 121 p. Disponible en: https://sightandlife.org/wp-content/uploads/2017/07/SAL_WFP_RiceFort_Situaci\%C3\%B3nactual.pdf

5. Ministerio de Desarrollo e Inclusión Social. Plan Multisectorial de Lucha Contra la Anemia [Internet]. Lima: Ministerio de Desarrollo e Inclusión Social; 2018 [Consultado 02 Jun 2019]. Disponible en: https://www.gob.pe/institucion/midis/informes-publicaciones/272499-plan-multisectorial-delucha-contra-la-anemia

6. Gil I. Propuestas para la mejora de la seguridad alimentaria de la población de Sarh [Tesis de licenciatura en Internet]. Madrid: Universidad Pontificia Comillas; 2014 [Consultado 2 Jun 2019]. 70 p. Disponible en: https://repositorio.comillas.edu/jspui/bitstream/11531/190/1/TFG000230.pdf

7. Rojas M, Suqui A. Conocimientos, actitudes y prácticas en la Administración de Micronutrientes por parte de las madres de niños menores de 3 años que acuden al Sub-Centro de Salud de Sinincay 2016 [Tesis de licenciatura de Internet]. Cuenca: Universidad de Cuenca; 2016. [Consultado 12 Jun 2019]. 87 p. Disponible en: http://dspace.ucuenca.edu.ec/handle/123456789/26108

8. Murrieta A. Factores que influyen en la adherencia al tratamiento de anemia ferropénica con micronutrientes en niños de 6 a 18 meses en el centro de salud Perú IV zona [Tesis de maestría en Internet]. Lima: Universidad César Vallejo; 2018 [Consultado 04 Sept 2019]. 121 p. Disponible en: http://repositorio.ucv.edu.pe/handle/20.500.12692/24493

9. Espichán P. Factores de adherencia a la suplementación con Sprinkles asociados al incremento de hemoglobina en niños de 6 a 60 meses, de asentamientos humanos del Distrito de San Martín de Porres [Tesis de licenciatura de Internet]. Lima: Universidad Nacional Mayor de San Marcos; 2013 $\left[\begin{array}{lllllll}\text { [Consultado } & 17 & \text { Oct } & 2019] & 43 & \text { p. } & \text { Disponible }\end{array}\right.$ https://cybertesis.unmsm.edu.pe/handle/20.500.12672/3417

10. Hinostroza F. Barreras y motivaciones en el nivel de adherencia a la suplementación con multimicronutrientes en madres de niños menores de 36 meses, Cercado de Lima [Tesis de licenciatura de Internet]. Lima: Universidad Nacional Mayor de San Marcos; 2015 [Consultado 12 Jun 2019]. 80 p. Disponible en: https://cybertesis.unmsm.edu.pe/handle/20.500.12672/4226

11. Pesantes $E$. Factores que influyen en el suministro de micronutrientes aa niños de 6 a 36 meses en el Centro de Salud Magllanal, Julio 2017 - Marzo 2018 [Tesis de maestría de Internet]. Chiclayo: Universidad César Vallejo; 2018 [Consultado 11 Sept 2019]. 63 p. Disponible en: http://repositorio.ucv.edu.pe/handle/20.500.12692/21731

12. Ministerio de Salud. Directiva Sanitaria No 056-MINSA/DGSP: Directiva sanitaria que establece la suplementación con multimicronutrientes y hierro para la prevención de anemia en niñas y niños menores de 36 meses [Internet]. Lima: Centro Nacional de Alimentación y Nutrición del Ministerio de Salud; 2014 [Consultado 12 Jun 2019]. Disponible en: http://bvs.minsa.gob.pe/local/MINSA/3933.pdf

13. Schauer C, Sunley N, Hubbell C, Nyhus C, Roca C, Tapia G, et al. Experiences and lessons learned for planning and supply of micronutrient powders interventions. Matern Child Nutr [Internet]. Febrero 2017 [Consultado 25 Abr 2020]; 120(2): 176-187. Disponible en: https://www.ncbi.nlm.nih.gov/pmc/articles/PMC5656916/

14. Ministerio de Salud. Norma técnica. Manejo terapéutico y preventivo de la anemia en niños, adolescentes, mujeres gestantes y puérperas [Internet]. Lima: Ministerio de Salud; 2017 [Consultado 21 Sept 2019]. Disponible en: http://bvs.minsa.gob.pe/local/MINSA/4190.pdf

15. Abelle E, Soto A, Muñoz V, Sanchez R, Carrera S, Pérez E, et al. Características de la prueba piloto: revisión de artículos publicados en enfermería [Internet]. Noviembre 2015 [Consultado 27 Abr 2020]. Disponible en: https://pesquisa.bvsalud.org/portal/resource/pt/biblio-1034782

16. Amezcua, M. Cómo estructurar un Proyecto de Investigación con prueba piloto. Gomeres [blog], 14 de Marzo 2015 [Consultado 04 May 2020]. Disponible en: https://www.fundacionindex.com/gomeres/?p=996

17. Thabane L, Ma J, Chu R, Cheng J, Ismaila A, Ríos L, et al. A tutorial on pilot studies: the what, why and how. BMC Med Res Methodol [Internet]. 06 Enero 2010 [Consultado 10 May 2020]; 1(2010): 1-10. Disponible en: https://bmcmedresmethodol.biomedcentral.com/articles/10.1186/1471-2288$10-1$ 
18. Junyong I. Introduction of a pilot study. The Korean Society of Anesthesiologists [Internet]. 14 Noviembre 2017 [Consultado 10 May 2020]; 70(6):601-605. Disponible en: https://europepmc.org/article/PMC/5716817

19. Muñoz C. Cómo elaborar y asesorar una investigación de tesis. 3era edición. México D.F: Pearson Educación; 2015. 488 p.

20. Hernández R, Mendoza C. Metodología de la Investigación. Las rutas cuantitativa, cualitativa y mixta. México: McGraw-Hill Interamericana; 2018. 713 p.

21. Burns N, Grove S. Investigación en Enfermería. 6ta ed. España: Elsevier; 2016. 554p.

22. Decreto Supremo que declara el Estado de Emergencia Nacional por las graves circunstancias que afectan la vida de la Nación a consecuencia del brote del COVID-19. Diario oficial del bicentenario El Peruano, $\mathrm{N}^{\circ}$ 044-2020-PCM.

23. Palomino J, Peña J, Zevallos G, Orizano L. Metodología de la Investigación: Guía para elaborar un proyecto en Salud y Educación. Lima: San Marcos; 2017. 223p.

24. Frías D. Apuntes de consistencia interna de las puntuaciones de un instrumento de medida [Internet]. España: Universidad de Valencia; 2019 [Consultado 01 Jun 2020]. 13 p. Disponible en: https://www.uv.es/ friasnav/AlfaCronbach.pdf

25. Sgreccia E. Manual de Bioética. Madrid: Biblioteca de autores cristianos; 2012. 970 p.

26. Insua J. Principialismo, bioética personalista y principios de acción en medicina y en servicios de salud. Persona y Bioética [Internet]. 06 Agosto 2018. [Consultado 15 May 2020]; 22(2): 223-426. Disponible en: http://www.scielo.org.co/pdf/pebi/v22n2/0123-3122-pebi-22-02-00223.pdf

27. Curo RL. Cumplimiento de la suplementación con multimicronutrientes a niños menores de 36 meses y su relación con los efectos secundarios y/o las creencias de los cuidadores en un establecimiento de salud del primer nivel de atención, Lima. 2018. [Tesis de licenciatura de Internet]. Lima: Universidad Nacional Mayor de San Marcos; 2019 [Consultado 04 Jun 2020]. 89 p. Disponible en: http://cybertesis.unmsm.edu.pe/handle/20.500.12672/10457

28. Samuel A, Putri N, Brouwer I, Adish A, Kebebe A, Osendarp S. Determinants of adherence to micronutrient powders among children 6-11 months of age in rural Ethiopia. Ann Nutr Metab [Internet]. Octubre 2017 [Consultado 30 May 2020]; 71(2): 404-405. Disponible en: https://www.karger.com/Article/Pdf/480486

29. Ibarra O, Morillo R. Lo que debes saber sobre la adherencia al tratamiento. Badalona: Sociedad Española de Farmacia Hospitalaria, Boehringer Ingelheim; 2017. 198 p. Disponible en: https://www.sefh.es/bibliotecavirtual/Adherencia2017/libro_ADHERENCIA.pdf

30. Poma R. Factores sociodemográficos y adherencia a la suplementación con multimicronutrientes en Centro Materno Infantil José Gálvez. [Tesis de maestría de Internet]. Lima: Universidad Cesar Vallejo; 2018 [Consultado 13 Jun 2020]. 121 p. Disponible en: http://repositorio.ucv.edu.pe/handle/20.500.12692/1621

31. Yanzapanta K, Tinoco A, Miranda K. Adherencia de la suplementación con multimicronutrientes y nivel de hemoglobina en niños de 6 a 36 meses del distrito de Huanca, Huancavelica, Perú, 2017. Revista Científica de Ciencias de la Salud [Internet]. 03 Diciembre 2018 [Consultado 15 Jun 2020]; 11(2): 40-47. Disponible en: https://revistas.upeu.edu.pe/index.php/rc_salud/article/view/1106

32. Mayca J, Medina A, Velásquez J, Llanos L. Representaciones sociales relacionadas a la anemia en niños menores de tres años en comunidades Awajún y Wampis, Perú. Rev Peru Med Exp Salud Pública [Internet]. 06 Septiembre 2017 [Consultado 22 Jun 2020]; 34(3): 414-422. Disponible en: https://www.redalyc.org/articulo.oa?id=36353391007

33. Ahmed F, Prendiville N, Narayan A. Micronutrient deficiencies among children and women in Bangladesh: progress and challenges. Journal of Nutritional Science [Internet]. 05 Septiembre 2016 [Consultado 24 Oct 2020]; 5(46): 1-12. Disponible en: https://www.ncbi.nlm.nih.gov/pmc/articles/PMC5465809/

34. Ildefonzo R, Uturunco N. Factores que influyen en la adherencia de multimicronutrientes en madres de niños de 6 -35 meses del Centro de Salud San Luis Lima - 2018. [Tesis de licenciatura de Internet]. Lima: Universidad Privada Norbert Wiener; 2017. [Consultado 17 Oct 2019]. 69 p. Disponible en: http://repositorio.uwiener.edu.pe/handle/123456789/2997

35. Mirkovic K, Perrine C, Subedi G, Mebrahtu S, Dahal P, Staatz C. Jefferds M. Predictors of micronutrient powder intake adherence in a pilot programme in Nepal. Public Health Nutr 
[Internet]. 26 Noviembre 2015 [Consultado 24 May 2020]; 19(10), 1768-1776. Disponible en: https://pubmed.ncbi.nlm.nih.gov/26708454/

36. López C. Factores asociados a la adherencia de la suplementación con multimicronutrientes en niños con anemia en la provincia de Tarata, 2017 [Tesis de licenciatura de Internet]. Tacna: Universidad Nacional Jorge Basadre Grohmann; 2017 [Consultado 04 Jun 2020]. 153 p. Disponible en: http://repositorio.unjbg.edu.pe/handle/UNJBG/3140

37. Carrión D. Factores que influyen en el consumo de multimicronutrientes, en niños(as) de 6 a 35 meses, Establecimiento de Salud Acora I - 4, Puno 2014. [Tesis de licenciatura de Internet]. Puno: Universidad Nacional del Altiplano; 2015 [Consultado 04 Jun 2020]. 103 p. Disponible en: http://repositorio.unap.edu.pe/handle/UNAP/1887

38. Bernal F, Rodríguez L, Díaz R. Estado nutricional, Desarrollo y Suplementación con Multimicronutrientes en niños de 6 a 36 meses, Inkawasi 2015. ACC CIETNA: Revista de la Escuela de Enfermería [Internet]. 14 Diciembre 2018 [Consultado 26 jun 2020]; 5(2), 39 - 43. Disponible en: http://revistas.usat.edu.pe/index.php/cietna/article/view/177

39. Oliveira C, Sampaio P, Muniz P, Cardoso M. Multiple Micronutrients in Powder Delivered Through Primary Health Care Reduce Iron and Vitamin A Deficiencies in Young Amazonian Children. Public Health Nutr [Internet]. 30 Mayo 2016 [Consultado 24 May 2020]; 19(16): 3039-3047. Disponible en: https://pubmed.ncbi.nlm.nih.gov/27237018/

40. Fernandes S, Cardoso M. Adherence to and acceptability of home fortification with vitamins and minerals in children aged 6 to 23 months: a systematic review. BMC Public Health [Internet]. 07 Abril 2016 [consultado 24 Oct 2020]; 16(299). Disponible en: https://bmcpublichealth.biomedcentral.com/articles/10.1186/s12889-016-2978-0

41. Orozco J, Vargas C, Rojas M, Herrera A, Montoya L, Sánchez J, et al. Efecto de los micronutrientes en polvo en el estado nutricional y en los valores hemáticos de preescolares sanos, Medellín, 2013. Revista de la Facultad Nacional de Salud Pública [Internet]. 15 Mayo 2015 [Consultado 26 Oct 2020]; 33(2):161-170. Disponible en: https://search.proquest.com/docview/1689569454/D0441A3B0E7B4522PQ/37?accountid=37610

42. Huamán L, Aparco J, Nuñez E, Gonzáles E, Pillaca J, Mayta P. Consumo de suplementos con multimicronutrientes Chispitas y anemia en niños de 6 a 35 meses: Estudio transversal en el contexto de una intervención poblacional en Apurímac, Perú. Rev Peru Med Exp Salud Pública [Internet]. Septiembre 2012 [Consultado 24 May 2020]; 29(3): 314-23. Disponible en: http://www.scielo.org.pe/scielo.php?script=sci_arttext\&pid=S1726-46342012000300004

43. Aparco J, Huamán L. Barreras y facilitadores a la suplementación con micronutrientes en polvo. Percepciones maternas y dinámica de los servicios de salud. Rev Peru Med Exp Salud Pública [Internet]. Noviembre 2017 [Consultado 18 Jun 2020]; 34(4): 590-600. Disponible en: https://scielosp.org/article/rpmesp/2017.v34n4/590-600/

44. Mamani N. Factores sociodemográficos y abandono del tratamiento de multimicronutrientes de niños de 6 a 36 meses, Centro de Salud Ollantay, 2017. [Tesis de licenciatura de Internet]. Lima: Universidad Inca Garcilaso de la Vega; 2017 [Consultado 04 Jun 2020]. 95 p. Disponible en: http://repositorio.uigv.edu.pe/handle/20.500.11818/2034

45. Livi M. Introducción a la demografía [Internet]. Barcelona: Editorial Ariel; 2007. [Consultado 13 Jun 2020]. 480 p. Disponible en: https://books.google.com.pe/books?id=5AW-asQX_MC\&printsec=frontcover\&dq=\%7DLivi+M.+Introducci\%C3\%B3n+a+la+demograf\%C3\%A $\mathrm{Da}+\% 5 \mathrm{BInternet} \% 5 \mathrm{D}$.+Barcelona:+Editorial+Ariel\&hl=es-

419\&sa =X\&ved=2ahUKEwin6ZvLptjsAhXWCrkGHTmqD8cQuwUwAHoECAAQBg\#v=onepage\&q\&f =false

\section{Editor}

Escuela de Enfermería de la Universidad Católica Santo Toribio de Mogrovejo, Chiclayo, Perú

\section{Cómo citar este trabajo}

Uceda-Vásquez N, Arriola-Arizaga M. Factores de adherencia a la suplementación con multimicronutrientes en niños menores de 36 meses de Monsefú, 2020. Acc Cietna: para el cuidado de la salud [Internet]. 2021; 8(2): 19 - 32. Disponible en: https://doi.org/10.35383/cietna.v8i2.604 


\section{Financiación}

El presente artículo no cuenta con financiación específica de agencias de financiamiento en los sectores público o privado para su desarrollo y/o publicación.

\section{Conflicto de interés}

Los autores del artículo declaran no tener ningún conflicto de intereses en su realización.

(c) Los autores. Este artículo es publicado por la Revista Acc Cietna: para el cuidado de la salud de la Escuela de Enfermería, Universidad Católica Santo Toribio de Mogrovejo.

Este es un artículo de acceso abierto, distribuido bajo los términos de la Licencia Creative Commons Atribución-NoComercial-CompartirIgual 4.0 Internacional (CC BY-NC-SA 4.0), que permite el uso no comercial, distribución y reproducción en cualquier medio, siempre que la obra original sea debidamente citada. 